PROCEEDINGS OF THE

AMERICAN MATHEMATICAL SOCIETY

Volume 126, Number 10, October 1998, Pages 3013-3017

S 0002-9939(98)04350-0

\title{
A NOTE ON SEQUENCES LYING IN THE RANGE OF A VECTOR MEASURE VALUED IN THE BIDUAL
}

\author{
BEGOÑA MARCHENA AND CÁNDIDO PIÑEIRO
}

(Communicated by Palle E. T. Jorgensen)

\begin{abstract}
Let $X$ be a Banach space. It is unknown if every subset $A$ of $X$ lying in the range of an $X^{* *}$-valued measure is actually contained in the range of an $X$-valued measure. In this paper we solve this problem in the case when we consider only vector measures of bounded variation.
\end{abstract}

\section{INTRODUCTION}

Let $X$ be a Banach space. It is still unknown if every subset $A$ of $X$ lying in the range of an $X^{* *}$-valued measure is actually contained in the range of an $X$-valued measure. In this paper we only consider vector measures of bounded variation, and solve this problem by exhibiting a sequence $\left(x_{n}\right)$ in the $\mathcal{L}_{\infty}$ Banach space $Y$ of Bourgain and Delbaen [BD] that lies in the range of a $Y^{* *}$-valued measure of bounded variation but is not contained in the range of a $Y$-valued measure with bounded variation. The proof is based in the following result that we prove in section 2 :

$A$ bounded sequence $\left(x_{n}\right)$ in $X$ lies inside the range of an $X^{* *}$-valued measure with bounded variation iff $\left(\alpha_{n} x_{n}\right)$ is contained in the range of an $X$-valued measure of bounded variation for every $\left(\alpha_{n}\right) \in c_{0}$.

We start by explaining some basic notation used in this paper. In general, our operator and vector measure terminology and notation follow [Ps] and [DU]. We only consider real Banach spaces. If $X$ is such a space, $B_{X}$ will denote its closed unit ball. The phrase "range of an $X$-valued measure" always means a set of the form $\operatorname{rg}(F)=\{F(A): A \in \Sigma\}$, where $\Sigma$ is a $\sigma$-algebra of subsets of a set $\Omega$ and $F: \Sigma \rightarrow X$ is countably additive.

\section{THE MAIN STEP}

For simplicity, we denote by $R_{b v}(X)$ the vector space of all sequences $\left(x_{n}\right)$ in $X$ lying in the range of an $X$-valued measure of bounded variation. We have obtained the following result.

Theorem 1. Let $\left(x_{n}\right)$ be a bounded sequence in $X$. The following statements are equivalent:

i) $\left(x_{n}\right) \in R_{b v}\left(X^{* *}\right)$,

Received by the editors October 14, 1996 and, in revised form, March 14, 1997.

1991 Mathematics Subject Classification. Primary 46G10, 47B10.

(C) 1998 American Mathematical Society 
ii) $\left(\alpha_{n} x_{n}\right) \in R_{b v}(X)$ for every $\left(\alpha_{n}\right) \in c_{0}$,

iii) $\left(\alpha_{n} x_{n}\right) \in R_{b v}\left(X^{* *}\right)$ for every $\left(\alpha_{n}\right) \in c_{0}$.

Proof. $i) \Rightarrow$ ii) Suppose $\left(x_{n}\right)$ is a sequence lying in the range of a vector measure valued in the bidual space $X^{* *}$ and having bounded variation. By [Pi1, Lemma 2] the operator $\Sigma e_{n}^{*} \otimes x_{n}: \ell_{1} \rightarrow X$ is integral (here $\left(e_{n}^{*}\right)$ is the unit basis of $\ell_{\infty}$ ). If $\left(\alpha_{n}\right)$ is a null sequence of real numbers, the operator $\sum e_{n}^{*} \otimes \alpha_{n} x_{n}: \ell_{1} \rightarrow X$ is the composition of $\Sigma e_{n}^{*} \otimes x_{n}$ with the diagonal compact operator $\left(\beta_{n}\right) \in \ell_{1} \rightarrow\left(\alpha_{n} \beta_{n}\right) \in$ $\ell_{1}$. So Grothendieck's Theorem [DU, p.252] tells us that $\Sigma e_{n}^{*} \otimes\left(\alpha_{n} x_{n}\right): \ell_{1} \rightarrow X$ is nuclear. Again it follows from [Pi1, Lemma 2] that $\left(\alpha_{n} x_{n}\right)$ belongs to $R_{b v}(X)$.

ii) $\Rightarrow$ iii) This is obvious.

iii) $\Rightarrow$ i) By hypotheses we can consider the linear map

$$
U:\left(\alpha_{n}\right) \in c_{0} \longrightarrow \sum_{n=1}^{\infty} e_{n}^{*} \otimes \alpha_{n} x_{n} \in I\left(\ell_{1}, X\right) .
$$

It is a standard argument to prove that $U$ has closed graph. Since $\ell_{1}^{*} \simeq \ell_{\infty}$ has the metric approximation property, $\mathcal{N}\left(\ell_{1}, X\right)$ is isomorphically isometric to a subspace of $I\left(\ell_{1}, X\right)[\mathrm{J}, \mathrm{p} .410]$. As $U$ maps each finite sequence $\left(\alpha_{1}, \alpha_{2}, \ldots, \alpha_{n}, 0, \ldots\right)$ into a nuclear operator, it is easy to prove that the rank of $U$ is contained in $\mathcal{N}\left(\ell_{1}, X\right)$. We also denote by $U$ the operator

$$
\left(\alpha_{n}\right) \in c_{0} \longrightarrow \Sigma e_{n}^{*} \otimes \alpha_{n} x_{n} \in \mathcal{N}\left(\ell_{1}, X\right) .
$$

Its dual operator $U^{*}$ takes $\mathcal{L}\left(X, \ell_{1}^{* *}\right)$ into $\ell_{1}$; in particular, $\mathcal{K}\left(X, \ell_{1}\right)$ into $\ell_{1}$. Let us prove that $U^{*}\left(\Sigma x_{n}^{*} \otimes e_{n}\right)=\left(\left\langle x_{n}, x_{n}^{*}\right\rangle\right)_{n}$ for every $T=\Sigma x_{n}^{*} \otimes e_{n} \in \mathcal{K}\left(X, \ell_{1}\right)$ (here $\left(e_{n}\right)$ is the unit basis of $\left.\ell_{1}\right)$. Using the trace duality, we obtain the following equalities:

$$
\begin{gathered}
U^{*}(T)_{m}=\left\langle u_{m}, U^{*}(T)\right\rangle=\left\langle U\left(u_{m}\right), T\right\rangle=\left\langle e_{m}^{*} \otimes x_{m}, T\right\rangle \\
=\operatorname{tr}\left(T \circ\left(e_{m}^{*} \otimes x_{m}\right)\right)=\left\langle\left(\left\langle x_{n}, x_{n}^{*}\right\rangle\right), e_{m}^{*}\right\rangle=\left\langle x_{m}, x_{m}^{*}\right\rangle
\end{gathered}
$$

for all $m \in \mathbb{N}$ and for all $T \in \mathcal{K}\left(X, \ell_{1}\right),\left(u_{m}\right)$ being the unit basis of $c_{0}$. To conclude the proof it suffices to notice that the adjoint of the operator

$$
U^{*}: \mathcal{K}\left(X, \ell_{1}\right) \longrightarrow \ell_{1}
$$

is defined by

$$
\left(\alpha_{n}\right) \in \ell_{\infty} \longrightarrow \Sigma e_{n}^{*} \otimes \alpha_{n} x_{n} \in I\left(\ell_{1}, X^{* *}\right),
$$

which can be proved in the same way using the trace duality. Then we have obtained that $\Sigma e_{n}^{*} \otimes x_{n}: \ell_{1} \rightarrow X^{* *}$ is integral and, therefore, so is $\Sigma e_{n}^{*} \otimes x_{n}: \ell_{1} \rightarrow X$.

If we consider countable additive vector measures, there is not an analogous theorem. To see this, let $X$ be a non-reflexive $\mathcal{L}_{\infty}$ space. By the non-reflexivity, there is a bounded sequence $\left(x_{n}\right)$ in $X$ that is not contained in the range of any $X^{* *}$-valued measure. Nevertheless, by [PR, Theorem 3.6] $\left(\alpha_{n} x_{n}\right)$ lies inside the range of an $X$-valued measure for every $\left(\alpha_{n}\right) \in c_{0}$.

Using the same method as in the proof of Theorem 1, we are going to prove a partial result in this general context. As in $[\mathrm{PR}]$, we denote by $R(X)$ the vector space of all sequences $\left(x_{n}\right) \in X$ lying in the range of a vector measure. If $\left(x_{n}\right)$ belongs to $R(X)$, we put $\left\|\left(x_{n}\right)\right\|_{r}=i n f\|m\|$, where the infimum is taken over all 
vector measures $m$ satisfying

$$
\left\{x_{n}: n \in \mathbb{N}\right\} \subset \operatorname{rg}(m) .
$$

$\left(R(X),\|\cdot\|_{r}\right)$ is itself a Banach space.

Proposition 2. Suppose $X$ is a Banach space satisfying the condition $\mathcal{K}\left(X, \ell_{1}\right) \subset$ $\Pi_{1}\left(X, \ell_{1}\right)$, and $\left(x_{n}\right)$ is a bounded sequence in $X$. The following statements are equivalent:

i) $\left(x_{n}\right) \in R(X)$,

ii) $\left(\alpha_{n} x_{n}\right) \in R(X)$ for every $\left(\alpha_{n}\right) \in c_{0}$,

iii) $\left(x_{n}\right) \in R_{b v}\left(X^{* *}\right)$.

Proof. First of all, notice that the inclusion $\Pi_{1}\left(X, \ell_{1}\right) \subset \mathcal{K}\left(X, \ell_{1}\right)$ holds for every Banach space $X$.

i) $\Rightarrow$ ii) This is obvious since the closed convex hull of a range is the range of another vector measure [DU, p.274].

ii) $\Rightarrow$ iii) In this part of the proof we follow the ideas of Theorem 1 . We consider the linear map

$$
U:\left(\alpha_{n}\right) \in c_{0} \longrightarrow\left(\alpha_{n} x_{n}\right) \in R(X) .
$$

It is continuos because its graph is closed.

If $S=\Sigma x_{n}^{*} \otimes e_{n} \in \Pi_{1}\left(X, \ell_{1}\right)$, by [Pi2, Proposition 2] the linear form

$$
\psi_{s}:\left(z_{n}\right) \in R(X) \longrightarrow \sum_{n=1}^{\infty}\left\langle z_{n}, x_{n}^{*}\right\rangle \in \mathbb{R}
$$

is well-defined, continuous, and $\left\|\psi_{s}\right\| \leq \pi_{1}(S)$. Then the linear map

$$
S=\sum_{n=1}^{\infty} x_{n}^{*} \otimes e_{n} \in \Pi_{1}\left(X, \ell_{1}\right) \rightarrow U^{*}\left(\psi_{s}\right) \in \ell_{1}
$$

is continuous. In the same way as in Theorem 1 we can prove that this map is defined by

$$
\Sigma x_{n}^{*} \otimes e_{n} \in \Pi_{1}\left(X, \ell_{1}\right) \rightarrow\left(\left\langle x_{n}, x_{n}^{*}\right\rangle\right) \in \ell_{1} .
$$

Since $K\left(X, \ell_{1}\right)=\Pi_{1}\left(X, \ell_{1}\right)$, dualizing again we have

$$
\left(\alpha_{n}\right) \in \ell_{\infty} \rightarrow \Sigma \alpha_{n} x_{n} \otimes e_{n}^{*} \in I\left(\ell_{1}, X^{* *}\right) .
$$

This shows that $\Sigma x_{n} \otimes e_{n}^{*}: \ell_{1} \rightarrow X$ is integral. So $\left(x_{n}\right) \in R_{b v}\left(X^{* *}\right)$.

iii $\Rightarrow$ i) In $[\mathrm{Pi1}]$ it is proved that every sequence in $\mathrm{X}$ lying in the range of a vector measure of bounded variation and valued in some superspace of $X$ actually belongs to $R(X)$.

In $[\mathrm{MR}]$ it is proved that the condition $K(X, Y) \subset \Pi_{1}(X, Y)$ implies that $\mathcal{L}(X, Y)=\Pi_{1}(X, Y)$, whenever $X$ or $Y$ has the bounded approximation property. So, we have the following equivalence:

$$
K\left(X, \ell_{1}\right) \subset \Pi_{1}\left(X, \ell_{1}\right) \Longleftrightarrow \mathcal{L}\left(X, \ell_{1}\right)=\Pi_{1}\left(X, \ell_{1}\right) .
$$

Recall that a Banach space $X$ is called a G.T.-space if $\mathcal{L}\left(X, \ell_{2}\right)=\Pi_{1}\left(X, \ell_{2}\right)[\mathrm{Ps}$. In $[\mathrm{G}$, Theorem 2] it is proved that the following statements are equivalent:

i) $\mathcal{L}\left(X, \ell_{1}\right)=\Pi_{1}\left(X, \ell_{1}\right)$,

ii) $\mathcal{L}\left(X^{*}, \ell_{1}\right)=\Pi_{1}\left(X^{*}, \ell_{1}\right)$.

From this equivalence the next result follows easily. 
Proposition 3. Let $X$ be a Banach space. The following statements are equivalent:

i) $\mathcal{L}\left(X, \ell_{1}\right)=\Pi_{1}\left(X, \ell_{1}\right)$,

ii) $X$ and $X^{*}$ are G.T.-spaces.

In fact, if a Banach space $X$ satisfies one of the two above conditions, then we have

$$
\mathcal{L}\left(X, \ell_{1}\right)=\Pi_{1}\left(X, \ell_{1}\right)=\Pi_{2}\left(X, \ell_{1}\right)
$$

\section{The COUNTEREXAMPLE}

Let $Y$ be the $\mathcal{L}_{\infty}$ space of Bourgain and Delbaen [BD]. They proved that $Y$ satisfies the Radon-Nikodým property and has a normalized unconditional basis $\left(e_{n}\right)$. We are going to show that $\left(e_{n}\right)$ lies inside the range of a $Y^{* *}$-valued measure of bounded variaton, but it does not belong to $R_{b v}(Y)$. In view of Theorem 1, if we prove that $\left(\alpha_{n} e_{n}\right) \in R_{b v}\left(Y^{* *}\right)$ for every $\left(\alpha_{n}\right) \in c_{0}$, then the sequence $\left(e_{n}\right)$ will belong to $R_{b v}\left(Y^{* *}\right)$. Notice that it suffices to consider null sequences $\left(\alpha_{n}\right)$ such that $\alpha_{n} \neq 0$ for all $n \in \mathbb{N}$. Take such a sequence $\left(\alpha_{n}\right)$. Since $Y$ is an $\mathcal{L}_{\infty}$-space, it follows from $[\mathrm{PR}]$ that $\left(\alpha_{n} e_{n}\right)$ lies in the range of a $Y$-valued measure. As $\left(\alpha_{n} e_{n}\right)$ is an unconditional basis of $Y$, by [AD] we have that $\left(\alpha_{n} e_{n}\right)$ is a weakly $\ell_{2}$-summable sequence. Then the map

$$
A:\left(\beta_{n}\right) \in \ell_{1} \longrightarrow \sum_{n=1}^{\infty} \beta_{n} \alpha_{n} e_{n} \in Y
$$

is absolutely summing because it admits the factorization $A=B \circ I, I$ being the inclusion map from $\ell_{1}$ to $\ell_{2}$ and $B$ the operator defined by $B\left(\beta_{n}\right)=\sum_{n=1}^{\infty} \beta_{n} \alpha_{n} e_{n}$. Now recall that absolutely summing and integral operators into an $\mathcal{L}_{\infty}$-space are the same [SR]. So, by [Pi1] the sequence $\left(\alpha_{n} e_{n}\right)$ lies in the range of a $Y^{* *}$-valued measure having bounded variation. Then we have proved that $\left(e_{n}\right)$ belongs to $R_{b v}\left(Y^{* *}\right)$. A final note in [Pi1] tells us that $\left(e_{n}\right)$ is actually in the range of a $Y$ valued measure. Therefore, $\left(e_{n}\right)$ is an unconditional basis of $Y$ lying in the range of a vector measure. Again a call to $[\mathrm{AD}]$ tells us that $\left(e_{n}\right)$ is a weakly $\ell_{2}$-summable sequence.

Finally, suppose $\left(e_{n}\right)$ is contained in the range of a $Y$-valued measure with bounded variation. Then the operator $T: \ell_{1} \longrightarrow Y$, defined by $T\left(\beta_{n}\right)=\Sigma \beta_{n} e_{n} \in Y$ for all $\left(\beta_{n}\right) \in \ell_{1}$, should be Pietsch-integral [Pi1]. Since $Y$ is a Radon-Nikodým space, it follows from [DU, Theorem VII.4.8] that $T$ is even nuclear. Nevertheless, $T$ is not a compact operator.

\section{REFERENCES}

[AD] R. Anantharaman and J. Diestel, Sequences in the range of a vector measure, Annales Societatis Mathematicae Polonae, Serie I: Comm. Math. XXX (1991), 221-235. MR 92g:46049

[BD] J. Bourgain and F. Delbaen, A class of special $\mathcal{L}_{\infty}$-spaces, Acta Math. 145 (1980), 155-176. MR 82h:46023

[DU] J. Diestel and J.J. Uhl, Jr, Vector measures, Math. Surveys 15, Amer. Math. Soc, Providence, R.I, 1977. MR 56:12216

[G] J.C. García-Vázquez, Tensor products of vector measures and sequences in the range of a vector measure, Proc. Amer. Math. Soc. 124 (1996), 3459-3467. MR 97f:46030

[J] H. Jarchow, Locally Convex Spaces, B.G. Teubner, Stuttgart, 1981. MR 83h:46008

[LR] J. Lindenstrauss and H.P. Rosenthal, The $\mathcal{L}_{p}$ spaces, Israel J. Math. 7 (1969), 325-349. MR 42:5012 
[MR] J.S. Morrel and J.R.Retherford, P-Trivial Banach Spaces, Studia Math. XLIII(1) (1972), 1-25. MR 47:2321

[Pi1] C.Piñeiro, Sequences in the range of a vector measure with bounded variation, Proc. Amer. Math. Soc. 123 (1995), 3329-3334. MR 96a:46033

[Pi2] C. Piñeiro, On p-summable sequences in the range of a vector measure, Proc. Amer. Math. Soc. 125 (1997), 2073-2082. MR 97i:46082

[PR] C. Piñeiro and L. Rodriguez-Piazza, Banach spaces in which every compact lies inside the range of a measure, Proc. Amer. Math. Soc. 114 (1992), 505-517. MR 92e:46038

[Ps] G. Pisier, Factorization of linear operators and geometry of Banach spaces, CBMS, vol.60, Amer. Math. Soc., Providence, R.I., 1986. MR 88a:47020

[SR] C.P. Steagall and J.R. Retherford, Fully nuclear and completely nuclear operators with applications to $\mathcal{L}_{1}$ and $\mathcal{L}_{\infty}$-spaces, Transac. Amer. Math. Soc. 163 (1972), 457-491. MR 54:3368

Departamento de Matemáticas, Escuela Politécnica Superior, Universidad de Huelva, 21810 La RÁBida, Huelva, Spain

E-mail address: candido@uhu.es 